\title{
Le lien familial en philosophie Patricia SIGNORILE
}

\author{
«Chaque famille secrète un ennui intérieur et \\ spécifique qui fait fuir chacun de ses membres quand il \\ lui reste un peu de vie. Mais elle a aussi une antique et \\ puissante vertu, qui reside dans la communion autour de \\ la soupe du soir, dans le sentiment d'être entre soi, et \\ sans manières, tels que l'on est - groupe de gens qui \\ sont entre eux tels qu'ils sont -.» \\ Paul Valéry Cahiers, T. II, «Homo, 1924, X, \\ 82», ed. La Pléiade, 1980, p. 1401
}

La famille ${ }^{1}$ se situe à l'intersection de facteurs biologiques, culturels et économiques. Sa fonction sociale - voire politique - est corrélative à une situation anthropologique et historique déterminée. L'importance de la famille du point de vue de l'identité et de la transmission, ainsi que ses incidences sociologiques et politiques, justifient son encadrement par le droit qui permet une évolution et une régulation harmonieuse du corps social et des liens entre les personnes.

Les philosophes n'ont pas manqué de s'intéresser à cette entité d'une façon qui, compte tenu du positionnement ontologique et critique de la discipline, peut paraître parfois déconcertante face aux règles du droit. Cependant l'une et l'autre sont en étroite adéquation. La réflexion philosophique, relative à l'être humain, démontre qu'il existe deux pôles qui balancent entre l'être soi et l'être social. À la lumière de l'oscillation qu'il suscite, le lien construit au sein de la famille en Occident relève-t-il d'une relation banale, fragile et révocable, d'une simple idéologie ou encore évolue-t-il dans une autre dimension?

Si Platon remet en question la propriété individuelle ainsi que le lien familial et la distinction entre les sexes, selon une organisation qui fait penser à une société communautaire, cette société repose sur une hiérarchie stricte fondée non pas sur une aristocratie de la naissance mais sur une aristocratie de l'intelligence. Pour Aristote, les hommes se regroupent tout d'abord en famille ou foyer puis en village, et enfin en cité, celle-ci n'étant rien d'autre que la communauté politique. La famille et le village subviennent à certains besoins de l'homme, mais c'est seulement dans la cité que l'autarcie individuelle est atteinte. L'individu isolé ne peut réaliser ses potentialités. L'homme est un animal politique et la société ou la cité sert de cadre de développement à ses dispositions naturelles.

Quant à Rousseau, à l'instar du précédent, il dissocie radicalement sphère privée et publique, point de vue qu'il partage avec Locke et Hobbes en admettant que le passage de l'état de nature à l'état social suppose une convention implicite passée entre les hommes. Avec le Contrat social, Rousseau refuse l'analogie entre famille et État, mais institue la distinction entre le domestique et le politique, la famille et la cité. Louis de Bonald considère, au contraire, que la famille se comporte comme « une petite société politique, hiérarchique et exemplaire » dont les membres forment une communauté indissociable.

Cf. Les rapports parents-enfants en quête de repères, Journée d'études organisée à Aix-en-Provence le 1er avril 2010, publié sous la direction de Emmanuel PUTMAN, Caroline SIFFREIN-BLANC, Jean-Philippe AGRESTI, 2011, PUAM ; Inventons la famille!, Françoise DEKEUVER-DEFOSSEZ, Philippe J EAMMET, Norbert ROULAND et Albert DONVAL, Les Éditions Bayard, collection: Société, Paris, 2001 ; Mort, naissance et filiation, Jean GUYOTAT, Paris, éditions Masson, 1980. 
Quoiqu'il en soit, ce lien qui est d'abord une réalité biologique qui précède et procède de chaque être humain, qui le constitue, désigne dans le vocabulaire courant aussi bien ce qui unit que ce qui entrave. Le lien familial en philosophie n'échappe pas à ces deux caractéristiques.

\section{Vie intime du philosophe et radicalisation théorique}

\subsection{Le lien conçu comme obstacle}

En effet, si la famille comme entité s'avère l'expression d'un lien naturel et culturel entre ses membres, elle n'en demeure pas moins caractéristique d'un lien subi. Elle naît d'un hasard et, par définition, implique dans la durée des échanges entre des personnes qui, peut-être, n'auraient pas forcément été partie prenante. Dans certains cas, elle produit des contraintes qui peuvent s'avèrer pesantes et des sources de conflits préjudiciables d'un point de vu personnel et social. S'en défaire implique alors des efforts considérables dans la mesure où les individus sont toujours conditionnés par les origines qui transmettent éducation, valeurs et souvenirs.

Une majorité de philosophes prennent de la distance par rapport au lien familial, car celui-ci est susceptible de fortement entraver la recherche de la vérité et surtout l'acquisition de la sagesse. Comme le constate Mathilde Lequin ${ }^{2}$, seul Socrate a transformé ironiquement le lien conjugal en une étape irrémédiablement vouée au bonheur, puisque le mariage en est la clé. En effet, avec « une bonne épouse, vous serez heureux» et avec « une mauvaise, vous deviendrez philosophe, ce qui est excellent pour l'homme. " Socrate était lui-même marié à Xanthippe, que la légende décrit comme redoutable. Diogène Laërce rapporte : "que Xanthippe en tonnant ferait aussi la pluie " ${ }^{3}$. Mais, selon Nietzsche, Socrate ne l'aurait épousée que pour montrer qu'il ne fallait pas se marier. En effet, un philosophe doit s'épargner le mariage, une femme étant une entrave, un obstacle, une source de problèmes de laquelle il faut rester éloigné. L'institution maritale empèche le philosophe d'atteindre un "optimum de conditions favorables, dans lesquelles il peut déployer toute sa force et atteindre le maximum de son sentiment de puissance $»$, de plus Nietzsche constate que Héraclite, Platon, Descartes, Spinoza, Leibniz, Kant, Schopenhauer n'étaient pas mariés.

Pourtant, Aristote, Hegel et Marx avaient convolé en justes noces mais, de l'avis de Jean-Marie Brohm', les contradictions internes - pour ces théoriciens de la dialectique - au sein d'un couple trouvent immédiatement à s'exporter sur le voisinage, provoquant à leur tour l'exacerbation des contradictions internes des couples amis et créant de cette façon une concaténation de contradictions et par consequent de litiges potentiels.

Ainsi, dans l'Antiquité, l'objectif prioritaire des philosophes concerne la recherche de l'autonomie individuelle et de la sérénité. Il est donc indispensable qu'ils se maintiennent à distance de tout ce qui peut compromettre la quête de l'ataraxie. Déjà Platon, dans la République, entreprend de faire évoluer l'oikos ${ }^{6}$ qui est le lieu d'un double attachement

\footnotetext{
« «a famille est-elle insupportable ? », Philo éditions - 2010 in Philosophie magazine, 045 (12/2010), p.3857.

Diogène LAËRCE, Vies et doctrines des philosophes illustres, Livre II, 36.

Frédéric NIETZSCHE, Contribution à la généalogie de la morale, L’Harmattan, Paris, février 2006, p. 229. Jean-Marie BROHM, Les principes de la dialectique, Eds de La Passion, 2003, p. 207.

Cf. Emile BENVENISTE, Le vocabulaire des institutions Européennes. Les éditions de Minuit, 1969, tome I chapitre 2, «Les quatres cercles de l'Appartenance sociale », page 308. Emile Benveniste constate que le sens premier d'oikos est celui d'une « grande maison groupant toute la descendance du chef de famille» et indique que la source du Grec « oikos» est issu du mot « woik», soit qui permet la désignation d'une fraction de l'unité sociale ou d'un clan, en indo Européen. À la même page l'auteur constate que le grec (w)ốkos occupe une situation intermédiaire. D'abord il s'agit d'une " (grande) maison » groupant toute la descendance du chef de famille, puis du substitut de dômos et «maison», «bâtiment» que l'on retrouve dans oikodômos, « constructeur, architecte » et de nombreux dérivés et composés. Par ailleurs, dans la langue de
} 
affectif, tourné d'une part vers les membres de la famille, et d'autre part vers la richesse accumulée dans et par l'oikos. Platon constate, qu'en l'état, cette force valorise le privé au détriment du commun, et se révèle par conséquent source de conflits. Pourtant celle-ci pourrait servir de lien si elle était transférée aux relations entre les citoyens dans la cité. Pour cette raison essentielle, l'économie doit être pensée à l'aune de la cité et non plus de l'oikos, ce qui permet l'expression du sentiment d'attachement qui doit réunir les citoyens de la cité juste. C'est donc avec des réserves que la famille est envisagée comme métaphore, puis modèle, des relations politiques.

Avec les Pères de l'église, le célibat va prendre une grande importance et accompagner les états mystiques. Le renoncement à la famille et à la lignée devient la condition d'un compagnonnage authentique avec Dieu. Saint-Augustin ne veut «entendre que la voix de Dieu ; (...) qu'il parle seul dans le silence universel, non avec les langues périssables de la chair (...) merveilleux instants de lumière et d'intelligence que Dieu accorde à nos soupirs, brillante et sainte image de l'éternelle béatitude !» Ces propos sont tenus en connaissance de cause, car Augustin avoue avoir connu "l'ardeur insensée du plaisir ». «À cette époque » note saint Augustin, «j'avais une femme; nous n'étions pas liés par les saints nouds du mariage. (...) mais je lui étais fidèle, et elle me l'était ; et cependant j'ai senti quelle différence il y avait entre cette union et celle du mariage». Il constate que "le mariage (est) fait en vue d'une parenté et d'une famille, tandis que dans l'union illégitime l'homme ne souhaite pas d'enfant, et pourtant il est forcé de les aimer aussitôt qu'ils sont nés ». Saint-Augustin a connu également les joies de la paternité et celles-ci l'ont comblé. Il écrit à ce propos que «Adeodat, (...) l'enfant de (son) péché, fut baptisé avec (lui) ». Celui-ci étant mort, désormais « (il) ne craint plus ni pour son enfance, ni pour sa jeunesse, ni pour son âge mûr (...)»7. À travers ces propos, on comprend que le lien familial est une grande source de joie mais qu'il développe une inquiétude récurrente incompatible avec les élans mystiques et la quête de la sérénité.

\section{2 Le premier mariage civil de l'histoire}

Par ailleurs, il est significatif qu'un grand nombre de philosophes aient opté pour le célibat, tout comme est significative la démarche de Rousseau, auteur d'un traité sur l'éducation, qui abandonne cependant systématiquement ses enfants auprès de l'Assistance Publique. De fait, les philosophes célibataires forment légion à l'âge classique, et si les penseurs des Lumières font souvent l'éloge de la famille comme institution essentielle de la société et ferment de la cohésion de la communauté, ils s'abstiennent paradoxalement d'avoir eux-mêmes « charge d'âmes ». Pour Voltaire «le mariage rend l'homme plus vertueux et plus sage » mais il reste conscient que "l'hymen et ses liens sont les plus grands ou des maux ou des biens. » En effet, « quel plaisir d'aimer publiquement, et de porter le nom de son amant! (Les) enfants, ces gages précieux, nés de l'amour, en sont de nouveaux næuds. (...) Mais tristement vendre par un contrat sa liberté, son nom, et son état, aux volontés d'un maître despotique, dont on devient le premier domestique ; se quereller ou s'éviter le jour (...) un tel hymen est l'enfer de ce monde $» .{ }^{.} \mathrm{C}$ 'est ainsi que Voltaire, sans

\footnotetext{
la poésie homérique, le terme oikos désigne aussi bien la " maison » d'un point de vue matériel que, par métonymie, «les habitants et les richesses qu'elle abrite». L'oikos représente la structure créée par une famille riche pour répondre à des exigences économiques et matérielles. Ulysse s'adressant aux Phéaciens dit : «dès que poindra l'Aurore, hâtez-vous pour me permettre (...) de fouler ma terre paternelle après tant d'épreuves subies. La vie peut me quitter, pourvu que je revoie seulement mes biens, mes servantes et le haut toit de ma grande maison », Odyssée, VII 222-5.

Cuvres de Saint Augustin, vol 13 - Les Confessions Livres I-VII, A. Solignac et E. Tréhorel et G. Bouissou, Desclée de Brouwer, 1980.

"L'hymen (mariage) et ses liens. Extrait de L'enfant prodigue, II, 2 - 1736, in CEuvres complètes de Voltaire, volume I, avec des notes et une notice sur la vie de Voltaire par Jean-Antoine-Nicolas de Caritat Condorcet (marquis de), Firmin-Didot Frères, 1843.
} 
enfant, ne s'est jamais marié, et que Rousseau épouse Thérèse Levasseur, après vingt-cinq ans de concubinage et l'abandon de leurs enfants.

En fait, pour ces penseurs qui élaboreront les bases théoriques de la démocratie moderne, le mariage et la famille paraissent sujet à controverse. Ils représentent en effet le poids des privilèges, de l'hérédité et de l'inertie sociale, qui entravent symboliquement la notion de progrès emblématique des Lumières. De plus, ces notions sont incompatibles avec le triomphe souhaité de la raison. Quant à Rousseau, il veut croire que "l'homme n'est pas fait pour le célibat», d'où un article de Jean Carbonnier, Il faut marier Rousseau, qui pose la question du mariage et de l'état civil des protestants français entre la révocation de l'édit de Nantes en 1685 et l'édit de tolérance de 1787. En effet, «Rousseau, protestant et genevois, apatride et étranger au royaume de France, fait du mariage un contrat, clé de voûte d'une religion civile, fondement même d'une laïcité ouverte aux protestants, aux jansénistes et à tous les inclassables. L'union de Rousseau avec Thérèse Levasseur prononcée en 1768 constitue le premier mariage civil de l'histoire de France »".

\section{3 L'impératif catégorique d'asexualité hors mariage}

Kant mène une existence de célibataire discipliné et abstinent, tout en valorisant le mariage du point de vu de l'appartenance sexuelle des corps. Dans la Doctrine du droit, le mariage est défini comme « la liaison de deux personnes de sexe different en vue de la possession réciproque, à vie, de leurs propriétés sexuelles ${ }^{10}$. \ L'intérêt pour le sexe relève par nature « de la conservation de l'espèce » et ne participe pas de la recherche du plaisir. La classification kantienne développée dans la Métaphysique des moeurs, au titre de la Doctrine du droit, distingue ainsi entre le « commerce sexuel naturel» et celui « contre nature ». L'homme peut user de ses organes sexuels de deux manières. La première consiste à suivre l'animalité qui est en lui - et Kant de préciser en latin : « vaga libido », « venus vulgivaga », 《 fornicatio », la seconde à prendre en compte sa part d'humanité. Dans ce cas, il suivra la loi, en l'espèce le mariage. Donc, pour Kant, la sexualité humaine en dehors du mariage n'est pas concevable, c'est même un « contrat nécessaire de par la loi de l'humanité » et de « par les lois de la raison pure ». Il s'agit d'un « impératif catégorique d'asexualité » hors du mariage, sachant que l'impératif catégorique" est la clé de voûte de la philosophie morale d'Emmanuel Kant. Kant condamne donc la sexualité et son économie bestiale hors du mariage. Ainsi, le concubinage est-il qualifié de « pactum turpe ». Celui-ci relève de l'extension et de la répétition dans le temps de la « location d'une personne pour un moment de jouissance », rien d'autre qu'un « pactum fornicationis ». Aussi le

\footnotetext{
- Jean-Jacques Rousseau ou paradoxes à propos du mariage cité par Monique COTTRET et Bernard COTTRET, Université de Paris Ouest-Nanterre-La Défense et Université de Versailles Saint-Quentin-enYvelines in Bibliothèque de la Société de l'Histoire du Protestantisme Français (B.S.H.P.F.) de 1979, « L'amour sans la loi », B.S.H.P.F. t. 125, p. 47-75 et t. 158/1 (janvier-mars 2012), p. 9-28.

Comme Diderot, Voltaire et bien d'autres écrivains, Rousseau choisit une femme du peuple pour partager son quotidien. C'est en accord avec Rousseau, qu'elle abandonne ses enfants à l'Assistance publique. Le 21 décembre 1790, l'Assemblée Nationale, " pénétrée de ce qu'elle doit à la mémoire de J.-J. Rousseau », décrète « il sera élevé à l'auteur d'Emile et du Contrat social une statue portant cette inscription La nation française libre à J.-J. Rousseau [...]. Marie-Thérèse Levasseur, veuve de J.-J. Rousseau, sera nourrie aux dépens de 1'Etat. » (Théroigne de Méricourt, Elisabeth Roudinesco, Seuil, 1989).

${ }^{\circledR}$ Souligné par Simone GOYARD-FABRE, p. 136, «Les figures du droit privé» in La Philosophie Du Droit De Kant, Vrin, 1996.

"Énoncé pour la première fois en 1785 dans Fondation de la métaphysique des mours, ce concept sera ensuite repris dans d'autres ouvrages d'éthique de l'auteur.
} 
concubinage $^{12}$ peut-il être assimilé à une sorte de prostitution mutuelle où chacun des contractants se sert de l'autre pour sa jouissance sensuelle et, partant, considère autrui exclusivement comme un moyen et non comme une fin, ce qui est, contraire à la morale kantienne. L'amour sexuel est donc l'amour «au sens le plus étroit du terme » et Kant le condamne moralement s'il n'est pas encadré par la loi du mariage.

Que la finalité du mariage d'après Kant soit la procréation apparaît comme une possibilité parmi d'autres, mais pour autant, rien n'indique la nature profonde du lien conjugal. En réalité, seul «un contrat, nécessaire de par la loi de l'humanité » et relevant des lois juridiques de la raison pure pratique, en indique le caractère «essentiel». D'ailleurs, cette posture sera vivement critiquée par Hegel pour lequel le mariage est le fruit du déterminisme et de la raison. Le lien contractuel lui est extérieur et contingent, tandis que le lien familial est absolu et immanent. La famille structure la «société civile » selon Les principes de la philosophie du droit.

\subsection{Une conception du mariage très conventionnelle}

Pour d'autres penseurs, c'est le lien ternaire père-mère-enfant qui se trouve mis en cause car il est loin de favoriser l'épanouissement individuel. Ces découvertes sont le fruit d'observations faites sur des patients, ainsi que par l'autoanalyse à laquelle se livre quotidiennement Freud ${ }^{13}$. Celui-ci est marié à Martha Bernays, qu'il épouse après quatre années de fiançailles et après lui avoir adressé près de 900 lettres. Freud adhère à une conception conventionnelle du mariage. Il écrit à sa future épouse, qu'ils sont «d'accord, je crois, toi et moi, pour estimer que la tenue du ménage, l'éducation des enfants et les soins à leur donner accaparent entièrement un être humain. $»^{14}$

Jean Blain constate qu' « un jour, il dissuade sa future femme d'aller rendre visite à une parente, enceinte avant son mariage; un autre, il lui interdit d'aller à la patinoire où un inconnu pourrait l'aider à se relever si elle venait à tomber et où il lui faudrait porter une jupe qui laisserait voir ses chevilles. Il se comportera de manière tout aussi conformiste avec ses six enfants (...) notamment (avec) les filles qui ne peuvent pas faire un pas hors de la maison sans être accompagnées, et dont les sorties, les fréquentations et les lectures sont l'objet d'un contrôle permanent $»^{15}$.

Freud, le médecin-philosophe fondateur d'une nouvelle discipline la psychanalyse, théoricien des névroses familiales et partisan résolu, en théorie seulement, de l'égalité des sexes, sera toute sa vie, dans son existence domestique, un bourgeois viennois parfaitement en osmose avec tous les préjugés de son temps. Freud qui considèrait que les liens tissés étaient susceptibles d'enfermement, générant et entretenant de puissantes névroses, les plus connues étant celles liées au Complexe d'Edipe, n'avait pas le don d'auto-critique. Il se soumettait avec docilité aux normes de son époque, tout en considérant que, la famille, oppressante, aliénante et rétrograde, représentait un obstacle majeur à l'épanouissement individuel. Pour autant qu'en est-il du lien qu'elle institue par le fait de l'irréductibilité de son principe d'existence?

\footnotetext{
12 D'après Jean MONTENOT («Le sexe selon Kant» in Lire 01 mai 2008), « aux yeux de Kant, il y a pire : les conduites " contre nature " qui sont hautement condamnables comme la zoophilie, l'homosexualité et même l'onanisme, " en totale opposition avec la nature humaine ». Dans ses Propos de pédagogie, publiés après sa mort, Kant conseille de présenter au jeune homme la masturbation « dans toute son abomination » en lui disant que " par là, il se rend inapte à la procréation de l'espèce », " qu'il attire sur lui une vieillesse précoce et que son esprit en subit de graves atteintes ». Dans sa Métaphysique des moeurs, il range la masturbation au nombre des comportements par lesquels l'homme ne respecte pas « son devoir envers luimême. »

13 Jean BLAIN, Lire, 01 novembre 2006, Lettre à Fliess du 15 octobre 1897.

"Claire METZ, Absence du père et separations, éditions L'Harmattan, 2009.

is Jean BLAIN, Lire, ibid supra.
} 


\subsection{Le lien familial conçu comme principe et modèle fondateur de la société politique}

Dans le deuxième chapitre du Livre I Du contrat social toutefois, Rousseau souligne que « la famille est (...) le premier modèle des sociétés politiques. » Mais il prend soin de nuancer cette affirmation en introduisant la notion de besoin. En effet si «la plus ancienne de toutes les sociétés et la seule naturelle est celle de la famille », le lien qui unit les enfants au père est subordonné à la notion de survie. Quand les enfants sont en mesure de subvenir seuls à leurs besoins, ce lien naturel disparaît. Il est alors, éventuellement, remplacé par un lien volontaire et la «famille elle-même ne se maintient que par convention ». Le passage de l'état de nature à l'état social, suppose donc une convention implicite passée entre les hommes. Cette séparation, qui dissocie radicalement sphère privée et sphère publique, Rousseau la partage avec Hobbes, pour qui «la famille comme l'état ne consiste pas seulement en une reunion d'êtres liés par l'intérêt et la concupiscence mais exige un consentement de l'enfant $\gg^{16}$, et Locke pour qui la famille existe avec le pouvoir paternel et la propriété privée avec le droit de propriété.

De plus, selon Hobbes le mariage peut être étendu aux ordres sacrés, à «ceux qui s'occupent continuellement de l'autel et de l'administration de l'Eucharistie », car " ils appellent donc la jouissance légitime des femmes défaut de chasteté et de continence, et ils font ainsi du mariage un péché, ou du moins une chose si impure et si sale qu'elle rend impropre au service de l'autel.» Or, "si la loi a été faite parce que la jouissance des femmes est de l'incontinence et qu'elle est contraire à la chasteté, alors tout mariage est vice, et si c'est une chose trop impure et trop sale pour un homme consacré à Dieu, alors, les activités naturelles, nécessaires, et quotidiennes, que tous les hommes font, devraient les rendre indignes d'être prêtres, étant encore plus sales. $»^{17}$

Si pour les philosophes, la famille - à travers les liens de la conjugalité et de la filiation - est à la fois une institution sociale, juridique et économique, selon l'anthropologue Claude Levi-Strauss, il en est de même dans toutes les sociétés humaines. À l'instar de la pensée de Durkheim, celle de Claude Lévi-Strauss a comme fondement un postulat primaire, soit qu'il n'y a pas d'institution ou de forme de la vie sociale qui reste limitée à l'instinct biologique. Dès lors, pour Lévi-Strauss, le groupe familial tire son origine de l'organisation sociale, plus spécialement du mariage. Il comprend le noyau constitué par le mari, la femme et les enfants nés de leur union. La particularité de l'institution familiale implique les règles que sont la prohibition de l'inceste et l'exogamie, l'atome de parenté et le mariage.

S'il est devenu banal de constater que la famille nucléaire, en Occident, connaît de profondes transformations depuis la fin des années 1950, la famille désigne toujours une communauté de personnes réunies par des liens de parenté, biologiques ou non, dotée d'une personnalité juridique contractuelle, d'un nom, d'un ou de plusieurs domiciles, d'un patrimoine commun qu'il soit culturel, numéraire ou foncier. Elle crée du fait de son existence une obligation juridique de solidarité morale et matérielle, étant supposée protéger et favoriser par l'éducation le développement social, physique et affectif de ses membres. Créant des liens, elle produit du sens par une sorte de volontarisme éducatif et affectif. Pour autant, est-il naturel d'être lié à sa famille?

La famille, que l'on croit naturelle, serait donc un fait de société, propre à l'homme. Le contrat en serait un outil majeur. Elle subit directement les changements de l'histoire et des mentalités et évolue de façon constante modifiant en cela les enjeux politiques qui la sous-tendent. Ainsi sa structure, autrefois tournée vers l'institution du mariage, propose-telle au $\mathrm{XXI}^{\circ}$ un changement de registre avec une grande diversité de mœurs, de comportements et de vocabulaire : pacs, monoparentalité, couples homosexuels, familles

\footnotetext{
${ }^{16}$ Thomas HOBBES, Leviathan, Des dominations paternelles et despotiques, chapitre 20, introduction, traduction et notes de François Tricaud, éditions Sirey, 1971.

"Ib. supra. Leviathan, chapitre 46, "Des ténèbres qui procèdent d'une vaine philosophie et de traditions fabuleuses ».
} 
recomposées, monoparentale, démariage... Pour autant, le groupe famillial, s'impose comme une donnée de la nature humaine, d'une exigence morale et vitale, et en cela relève d'un processus normatif soumis toutefois aux contingences anthropologiques et historiques. En ce sens, le lien qui en découle revêt une fonction sociale unificatrice.

À la fois institution et système de relations, la famille relève de l'univers individuel et collectif. Il s'agit du premier espace social auquel est confronté l'individu et qui délimite l'espace de ses possibles. À partir de cet espace, s'opère l'expérience de la subjectivité qui, sur la durée, va modeler, altérer voire effacer les liens initiaux - excepté celui de la transmission de l'identité donnée par le nom (ou de l'identité de substitution pour les enfants nés sous "X") - que la nature présuppose. C'est, en partie, pour cette raison que la famille s'appuie sur des fondements juridiques et normatifs précis, capables de s'adapter aux bouleversements et soubresauts imposés par la sociologie et l'histoire.

L'intrication de cette permanence et d'un coefficient d'adaptabilité élevé invitent les philosophes à s'interroger sur la spécificité du lien familial qui du coup revêt une certaine polysémie. En effet, pour une majorité d'individus, la reproduction puis la socialisation des enfants ne sont plus une fin en soi, et le lien familial est porteur d'enjeux politiques et idéologiques. Il est soumis à un principe d'accomodation permanent, en ce sens qu'il évolue en fonction des choix et des contraintes de chacun. L'histoire individuelle et collective se construit en même temps qu'elle est subie.

D'autre part, la notion de lien désigne les relations intrafamiliales, mais également celles qui existent entre famille et société, et intervenant à la fois dans les domaines domestiques, affectif et financier, désigne fréquemment la parenté au sens large. Cette notion de famille élargie est valorisée par les acteurs sociaux et politiques, puisque un tel réseau de solidarité, même fondé sur des principes incertains et fluctuants, est susceptible de prendre le relais d'un Etat qui ne peut plus être providentiel. Elle met de plus en exergue une des composantes majeures du lien familial, constituée par le principe de transmission et les solidarités naturelles entre générations ${ }^{18}$.

\section{La famille entre disposition naturelle et contrainte : les enjeux du lien}

\subsection{De Rousseau à Engels, la remise en question.}

Dans le Contrat social, livre premier - la notion de contrat étant essentielle au siècle de la Raison -, Rousseau refuse l'analogie entre famille et État, et pose la dissimilitude entre les domaines domestique et politique, entre famille et Cité. «La famille est donc si l'on veut le premier modèle des sociétés politiques, le chef est l'image du père, le peuple est l'image des enfants, et tous étant nés égaux et libres n'aliènent leur liberté que pour leur utilité. Toute la différence est que dans la famille l'amour du père pour ses enfants le paye des soins qu'il leur rend, et que dans l'État le plaisir de commander supplée à cet amour que le chef n'a pas pour ses peuples». La dimension affective occupe chez Rousseau une place déterminante dans la singularisation de la famille, bien que celle-ci se maintienne par convention, c'est-à-dire par l'accord implicite de ses membres. La volonté prend le relais de la nature, et transforme la famille en réalité politique.

C'est dans cette perspective qu'au XIX' siècle, le mode de vie bourgeois s'affirme, tout en étant perçu comme répétitif, monotone, dépourvu d'enthousiasme. Il entraîne un phénomène de rejet chez les penseurs et plus largement les intellectuels. La famille et le mariage qui en est l'un des éléments essentiels, font l'objet de critiques. "La famille me

\footnotetext{
". Sous la direction de Georges EID, La famille, le lien et la norme, éditions L’Harmattan, mars 1998.
} 
paraît quelque chose d'assez étroit et d'assez misérable », note Flaubert dans ses Souvenirs, notes et pensées intimes. Arthur Schopenhauer est formel «les mariages d'amour sont conclus dans l'intérêt de l'espèce et non au profit de l'individu. "Quant à Søren Kierkegaard, il note, dans les Étapes sur le chemin de la vie, que "le célibataire peut risquer plus dans le monde de l'esprit que le marié», puisqu'il peut «tout mettre en jeu en se souciant seulement de l'idée». Il délaisse sa fiancée afin de sauvegarder sa vision idéale du mariage et constate que «le mariage est et restera le voyage de découverte le plus important que l'homme puisse entreprendre ; toute autre connaissance de l'existence, comparée à celle d'un homme marié, est superficielle, car lui et lui seul a vraiment pénétré l'existence.»

L'avis de Friedrich Nietzsche' ${ }^{19}$ est sans appel, ni équivoque possible. Il constate l'effondrement de toutes les traditions ainsi que de leurs corrolaires les institutions. C'est " le mariage d'amour» qui a provoqué l'effondrement de l'instinct de propriété. En effet, «nos institutions ne valent plus rien : là-dessus tout le monde est d'accord. Pourtant la faute n'en est pas à elles, mais à nous. Tous les instincts d'où sont sorties les institutions s'étant égarés, celles-ci à leur tour nous échappent, parce que nous ne nous y adaptons plus. De tous temps le démocratisme a été la forme de décomposition de la force organisatrice (...). Pour qu'il y ait des institutions, il faut qu'il y ait une sorte de volonté, d'instinct, (...) une volonté de tradition, d'autorité, de responsabilité, établie sur des siècles (... ). Lorsque cette volonté existe, il se fonde quelque chose comme l'imperium Romanum (...). Tout l'Occident n'a plus ces instincts d'où naissent les institutions, d'où naît l'avenir : rien n'est peut-être en opposition plus absolue à son " esprit moderne ».

Nietzsche constate que dans «(...) le mariage moderne apparemment toute raison s'en est retirée : pourtant cela n'est pas une objection contre le mariage, mais contre la modernité. La raison du mariage - elle résidait dans la responsabilité juridique exclusive de l'homme (...) dans le principe de son indissolution, (...) dans la responsabilité des familles quant au choix des époux. Avec cette indulgence croissante pour le mariage d'amour on a éliminé les bases mêmes du mariage, tout ce qui en faisait une institution. (...) On ne fonde pas le mariage sur "l'amour ", on le fonde sur l'instinct de l'espèce, sur l'instinct de propriété (la femme et les enfants étant des propriétés), sur l'instinct de la domination qui sans cesse s'organise dans la famille en petite souveraineté, qui a besoin des enfants et des héritiers pour maintenir, physiologiquement aussi, en mesure acquise de puissance, d'influence, de richesse, pour préparer de longues tâches, une solidarité d'instinct entre les siècles. " Et de conclure que désormais, «le mariage est complètement dépourvu de sens. Le mariage moderne a perdu sa signification, par conséquent on le supprime ${ }^{20} . »$

Mais s'il villipende désormais le mariage, qui propose une formule démocratique du bonheur, celui-ci pour autant convient à la masse. Quant à l'idéal ascétique du philosophe dans la Généalogie de la morale, il affirme que "la fécondité de ce genre d'esprits se manifeste autrement que par sa progéniture ».

\subsection{Les trois étapes de l'invention du lien familial}

De leur côté, avec une vision plus sociologique, Engels et Marx souhaitent libérer les individus à la fois de leur nature et des contraintes liées à celle-ci, en particulier des contraintes familiales. Dans le contexte évolutionniste de leurs théories, il est fondamental que l'homme soit libre de tout impératif biologique. L'humain est créateur de sa propre

19 Friedrich NIETZSCHE, Le Crépuscule des idoles, § 39

${ }^{20} \mathrm{Ib}$. supra. 
histoire et rien ne peut en limiter ou en diriger l'évolution ${ }^{21}$. Dans l'Idéologie allemande, ils soutiennent que la famille est fondatrice du lien social et contribue au principe de la division du travail. Elle possède une histoire étroitement dépendante des conditions économiques. D'ailleurs, dans L'origine de la famille, de la propriété privée et de l'Etat, Engels affirme élucider, scientifiquement, le rapport entre les deux instances, celle de la production et celle de la famille.

Engels s'appuie sur les trios phases de l'époque pré-capitaliste définies par Morgan et qui sont, la sauvagerie, la barbarie et la civilisation. Il montre comment, à chacune de ces étapes, s'impose un type de famille particulier, adapté aux impératifs de la production. Dans la société gentilice, c'est la famille punaluenne qui domine. Des soeurs peuvent être les femmes communes de maris communs. Mais, les soeurs et les frères ne peuvent entretenir de commerce sexuel. La filiation est exclusivement feminine. L'économie est domestique. Dans la famille «appariée », l'homme vit avec une femme, cependant l'infidélité est un droit. Les enfants appartiennent strictement à la lignée maternelle.

Avec le développement de la production, de l'échange et de la propriété privée, la famille conjugale monogame apparaît et elle doit son origine à la famille « appariée ». Le père transmet alors son héritage. Dans l'analyse marxiste, les liens familiaux ont une origine économique qui se caractérise par la suprématie de l'homme envers la femme. Karl Marx et Friedrich Engels affirment, dans Le Manifeste du Parti communiste que "la bourgeoisie a déchiré le voile de sentimentalité qui recouvrait les relations de famille et les a réduites à n'être que de simples rapports d'argent ». La véritable famille conjugale, celle qui repose sur des rapports d'estime et d'affectivité, n'existe que dans le prolétariat, car "il ne s'y trouve aucune propriété... (et) il manque tout stimulant pour faire valoir la suprématie masculine...».

Pour Engels, si la famille monogame n'a pas à disparaitre en tant que telle, elle doit cependant cesser d'être l'unité économique de la société. En revanche, Alexandra Kollontaï a anticipé la dissolution de la famille. D'une part, parce que celle-ci n'aurait plus pour fonction la conservation des richesses familiales et, d'autre part, parce que les obligations familiales seraient transférées à la charge de la société et de l'Etat. Pour cette philosophe "la famille telle qu'elle existe encore vit ses derniers jours et elle est irrémédiablement condamnée à périr avec la société de classes antagonistes». Ainsi, «les influences sociales sont si complexes, leur action si diversifiée, qu'il est actuellement impossible d'imaginer avec précision ce que sera la forme dans laquelle se mouleront, après un changement radical de toute la structure de la société, les rapports conjugaux de l'avenir. »

Quoiqu'il en soit, Engels est le premier philosophe marxiste à analyser l'évolution de la famille à partir des positions du matérialisme historique. La famille s'apparente à une catégorie historique en lien organique entre ses formes, les étapes du développement et de l'économie de la société. Engels fait coïncider la diminution de l'influence des liens de parenté avec la victoire de la propriété privée. Alors «le règne de la famille est complètement dominé par le régime de la propriété » et il soumet à une vive critique la famille bourgeoise. Ce n'est que grâce à la société socialiste, que les femmes seront associées à la production sociale et qu'une véritable égalité avec les hommes sera instaurée, les femmes étant affranchies des soucis du ménage que la société assumera de façon croissante. C'est seulement dans cette étape que s'affirmera un type de famille nouveau fondé sur l'égalité des sexes, le respect mutuel et l'amour.

${ }^{2}$ Cf. Friedrich ENGELS, Origine de la famille, de la propriété et de l'Etat, Paris, Editions Sociales, 1972 (1 re éd. en allemand en 1884).

22 Alexandra KOLLONTAÏ, Marxisme et Révolution sexuelle, François Maspéro, Petite collection maspéro, 1973. 
Johann Jakob Bachofen ${ }^{23}$, juriste, philologue et sociologue suisse, théoricien du matriarcat, justifie celui-ci par des preuves puisées dans de nombreux passages de la littérature classique de l'Antiquité. Dans un premier temps, il a inspiré Engels qui par la suite s'en est éloigné. D'après lui, l'humanité a d'abord vécu dans des rapports sexuels dépourvus de toute règle, qu'il désigne par le terme d' "hétaïrisme ». Excluant toute paternité certaine, le type de filiation qui en découle ne peut qu'être attribué en lignée féminine, selon le droit maternel. Cela fut le cas pour tous les peuples de l'antiquité. Les femmes, en tant que mères, sont les seuls parents légitimes de la lignée, et bénéficient en retour de respect et de prestige qui, selon la conception de Bachofen développent la «gynécocratie ». Le passage au mariage conjugal, où la femme n'appartient qu'à un seul homme, enterine un antique commandement religieux qui condamne le droit traditionnel des autres hommes à la même femme. Cette pratique fautive doit être expiée, par la femme ui s'offre ponctuellement à d'autres hommes.

Mais, Engels et les évolutionnistes ne furent pas les seuls à considérer le mariage monogamique et la famille restreinte, comme structure sociale liée à l'état de développement économique, politique et social des sociétés avancées. En 1892, Durkeim considére que la famille fondée sur le lien conjugal est issue des sociétés germaniques, c'est-à-dire des sociétés qu'il juge comme étant les plus civilisées en cette fin du XIX ${ }^{\circ}$ siècle. Vers 1950, Talcott Parsons ${ }^{24}$ reprend ce raisonnement à son compte et l'applique à la société américaine. De fait, même s'il est toujours possible de discuter de l'aspect idéologique, la vision épistémologique d'Engels s'est avérée valable, dans ce sens que l'idée d'évolution a été globalement admise, et que le lien entre, d'une part, le niveau économique, social et politique d'une société et, d'autre part, le lien familial est aujourd'hui avéré. Cette vision renforce les idées d'Auguste Comte, qui proposait une vision «positive» et «scientifique» de la famille, mettant en évidence le fait que la famille est une réunion de relations et de statuts. Fondée sur un principe hiérarchique, la famille est ainsi essentielle dans le processus de socialisation de l'individu et inséparable de sa relation avec la communauté et l'Etat's .

Ainsi, la position des philosophes à l'égard de la famille démontre bien que le lien familial est complexe et multiple. Tantôt il est rejeté, d'autres fois il sert de modèle épistémologique. Pour autant, il semble exister une sorte d'historicité inaliénable de celui-ci.

\subsection{L'historicité inaléniable du lien familial}

En 1960, dans son livre L'Enfant et la vie familiale sous l'Ancien Régime, Philippe Ariès initie une approche historique de l'enfance mais aussi du lien familial. Par la suite, ses études ont été affinées à l'aide de relevés des naissances, baptêmes, décès, mises en nourrices, et ont ainsi permis de reconstituer non seulement les compositions des structures familiales mais aussi celles des mœurs. Les sources qu'il utilise sont diverses : registres paroissiaux, données littéraires et iconographiques, correspondances privées et officielles entre agents de l'État, commentaires publics et textes de lois. Toutes ces données concrètes montrent que les manifestations d'attachements des parents envers leurs enfants sont variables suivant les périodes et suivant les classes sociales. Par exemple, aux XVIIème et XVIIIème siècle, de très nombreux enfants de la noblesse et de la bourgeoisie, et de toutes couches sociales en milieu urbain, sont envoyés en nourrice, loin de leur parents, et sont traités avec une certaine négligence par ces nourrices « mercenaires ». Ces traitements

\footnotetext{
${ }^{23}$ Johann Jakob BACHOFEN, Le Droit Maternel, recherche sur la gynécocratie de l'Antiquité dans sa nature religieuse et juridique, trad. Étienne Barilier, éd. L'Age d'Homme, 1996.

${ }_{24}$ Talcott PARSONS a élaboré un système de pensée reposant sur deux notions essentielles : celle d'action, objet principal de l'analyse sociologique, et celle de valeur, corrélat nécessaire de la première. Il retrouve non seulement la notion de valeur chez Weber, mais aussi dans la théorie de Durkheim sur la religion et chez Pareto. Tout système social doit, pour exister et se maintenir, répondre à quatre impératifs fonctionnels : la poursuite de buts (goal attainment), l'adaptation au milieu environnant, l'intégration des membres dans le système social, la stabilité normative ou latente (pattern maintenance).

${ }_{2}$ Auguste COMTE, Système de Politique Positive, 1851.
} 
augmentent la mortalité infantile de manière importante, à tel point que certains représentants de l'État, inquiets pour la santé publique et le nombre des futurs travailleurs, constatent que les parents se préoccupent moins de leurs enfants que de la santé de leurs chevaux. Au XVIIIème siècle, s'amorce une baisse de la mortalité infantile grâce, sans doute aux progrès de la médecine et de l'hygiène et aux soins accordés par les parents. C'est au XIXème siècle que le recours aux nourrices tombe en désuétude au profit de manifestations d'amour maternel qui se généralisèrent (mais le rôle affectif des pères semble en général bien différent).

À la lumière de ce travail, Edward Shorter et Élisabeth Badinter, entre autres, considèrent que l'amour maternel, ciment du lien familial est un sentiment moderne, construit socialement avec le concours de l'État pour répondre à des intérêts multiples. Élisabeth Badinter estime que la femme n'est pas, par sa nature, plus susceptible que l'homme d'obligations de soins et d'amour envers son enfant. D'autres historiens, comme Marie-France Morel $^{26}$, considèrent que les comportements observés « ont leur rationalité et (...) peuvent exprimer un amour différent du nôtre », que "les enfants ont toujours été aimés, d'une manière ou d'une autre, sinon ils n'auraient pas survécu. » Soulignant les inévitables lacunes des informations disponibles, elle considére qu' « à certains moments, les mères et les nourrices du peuple sont les seules à les aimer, et ces sentiments des humbles laissent peu de traces dans nos sources ». Comme le rappelle Irène Théry, «c'est le quotidien partagé, l'exercice des responsabilités éducatives par le parent, les échanges affectifs entre lui et l'enfant qui tissent le lien de la filiation. ${ }^{27}$

Quoiqu'il en soit, l'identité personnelle se structure sur la base d'habitudes incorporées qui se transforment en automatismes afin d'éviter la remise en question de l'organisation familiale et les risques de l'anomie. Par consequent, les êtres humains, loin de naître sociaux, le deviennent grâce à la force économique et socialisante des systèmes politiques, culturels, religieux, contingents et arbitraires dans lesquels ils sont immergés. La famille occupe dans ce dispositif une place importante car elle est l'institution susceptible de lier les individus indépendamment de leur volonté et de leur intérêt.

Aucun discours sur la famille ne pourrait en épuiser le sens. Elle ne saurait être rationalisée sans être dénaturée. En dépit des mutations sociétales, la famille conserve, en règle générale, un rôle préponderant dans la construction du lien social, source de solidarité, d'ouverture, d'humanité, de soutien. Comme l'écrit Pierre Centlivres, «dans les institutions matrimoniales que se donnent les peuples, le biologique est toujours subordonné, ou plutôt réinterprété, par l'ordre culturel $\gg^{28}$. Elle a pour caractéristique d'être établie par une parole d'adoption, que ce soit a priori, dans le cadre du mariage, en vertu de ce que les juristes appellent « la présomption de paternité légitime », ou a posteriori, en dehors du cadre du mariage, par l'effet d'une reconnaissance ponctuelle de paternité/maternité. La filiation ne peut faire l'économie de cette parole de reconnaissance. La nomination n'est-elle pas, en soi, une sorte de résumé symbolique, ou de condensé, du lien familial?

\footnotetext{
${ }_{26}$ Encyclopadia Universalis, « Histoire de l'enfance ».

27 Irène THERY, « Différence des sexes et différence des générations », revue Esprit, Paris, Le Seuil, 1996.

${ }^{2}$ Pierre CENTLIVRES, «Amis, amants, mariage...un point de vue anthropologique », in La reconnaissance des couples homosexuels (Genève: Labor \& Fides, 2000), p. 57.

Selon Pierre Centlivres, « si l'on fait le tour d'horizon des sociétés humaines, le mariage apparaît comme une institution à plusieurs faces (...): selon un axe vertical, celui de la reproduction de la société, il légitime l'appartenance de l'enfant, né du couple, et l'assigne au groupe du père ou de la mère, et selon un axe horizontal, il établit des relations d'alliances, par-delà le lien époux-épouse, entre deux groupes de parents. » Op. cit. p. 58. Comme le dit le doyen Jean Carbonnier, «ce qui donne aujourd'hui au mariage sa portée juridique, c'est ce que les juristes appellent la 〈présomption de paternité légitime〉, c'est-à-dire que les enfants issus d'une femme mariée sont présumés être le fruit du mari. Ainsi pour définir juridiquement le mariage, il ne faut pas penser d'abord au couple, mais aux enfants possibles, et à leur rattachement effectif au mari de la mère. » «L'évolution contemporaine des mœurs », in Fac étude (Vaux-sur-Seine : FLTE, no 16, avril 1990), p. 8. Mais aussi cf. Vous avez dit famille ? Le lien familial à définir en matière d'inceste, mercredi 29 février 2012, 18:38, Mélina DOUCHY-OUDOT, http://www.placedeleglise.fr/
} 


\section{Conclusion}

Ainsi, même si les philosophes anciens et modernes sont considérés pour s'être préoccupés en priorité d'objectifs introspectifs, nombreux sont ceux qui ont envisagé la conjugalité, l'identité, la filiation, entre famille et politique, entre sphère privée et sphère publique, entre phénomène économique et culturel.

La famille se trouve au centre des problématiques philosophiques et sociétales, parce qu'elle se situe à la frontière de l'individuel et du collectif en même temps qu'elle s'en démarque. Face à la montée en puissance de l'individualisme et de la précarité sociale que connait la société contemporaine ${ }^{29}$, mais également face à l'évolution des valeurs, les normes qui semblent paradoxalement s'imposer au sein de la communauté familiale, sont -en règle générale - celles du don, de l'échange et du libre arbitre, de l'irréductibilité du lien entre les personnes mais aussi de modèles nouveaux en émergence ${ }^{30}$.

Désormais, dans l'Occident contemporain, le modèle quelque peu réducteur et archétypal de la famille s'est transformé, même si celui-ci continue à inspirer les modèles organisationnels, les systèmes de pensée et les idéologies. Le lien familial s'inscrit donc à l'intérieur d'une réalité évolutive complexe. Le transfert des responsabilités et des fonctions, opéré tout au long des siècles, a été relayé ou supplanté au fil du temps par d'autres institutions. Aujourd'hui, l'obsession de la filiation est compatible avec la dissociation des fonctions parentales et éducatives, la spécialisation des rôles, la socialisation des activités qui caractérisent un autre type d'organisation sociale. Les mœurs, les normes, les lois, les philosophes et autres spécialistes, réfléchissent, discernent, nomment, caractérisent, encadrent, la mutation des liens. Le lien familial se rapprocherait de l'idéal du lien social, c'est-à-dire qu'il serait en definitive fondé sur l'égalité, le libre consentement et le contrat ${ }^{3}$.

Mais c'est ainsi le droit - philosophie pratique en actes - qui dans son évolution temporelle organise le complexe et le compliqué de la parenté comme construction sociale, et dans les cas de défaillance fixe les bornes et les repères, indique les interdits, les devoirs, les obligations, les droits des individus les uns par rapport aux autres. En définitive, il « institue la vie » selon l'expression de Pierre Legendre et contribue aux grands principes de l'eudaimonia: des philosophes grecs à travers le changement des mentalités.

\footnotetext{
${ }^{2}$ Hélène BELLEAU, Être parent aujourd'hui : la construction du lien de filiation dans l'univers symbolique de la parenté, INRS-Urbanisation, Culture et Société, http://www.erudit.org/revue/efg/2004/v/n1/008891ar.html

${ }_{30}$ Relations avec les beaux-parents dans le cas de mariages multiples par exemple (ressources sociales, financières, éducatives), nouvelles méthodes de procréation et leur impact sur la famille, homosexualité et homoparentalité, mariage et famille, famille et immigration...

"Cf. Corinne RENAULT-BRAHINSKY, L'essentiel du droit de la famille, 6ème edition, Etude (poche), 2011. L'incursion du contrat dans le droit de la famille pose aujourd'hui la question de la survivance de la dimension institutionnelle de la famille. Cette contractualisation du droit de la famille a notamment pour source la conception individualiste du couple développée par la cour européenne des droits de l'homme qui prône un pluralisme des moeurs. Le développement de l'autonomie des individus s'est faite au détriment da la puissance maritale et étatique et s'est affirmée au travers des principes d'égalité et de liberté. Ce retrait du droit de l'institution familiale s'est accompagné d'une mise en avant du contrat, qui trouve sa définition à l'article 1101 du code civil. Il est l'expression même de cette place abandonnée à la liberté individuelle.

32 Cf. Alasdair MacINTYRE, Quelle justice? Quelle rationalité ?, trad. Michèle Vignaux d'Hollande, PUF, 1993, p.111. Chez les Grecs, la justice (dikaiosunè) est une norme de la nature. Le juste ordonnancement de la nature et l'organisation de la polis sont adossés au bonheur (eudaimonia) et à l'épanouissement humain dont le caractère suprême est un climat de paix.
} 